\title{
Neurally adjusted ventilatory assist decreases work of breathing during non- invasive ventilation in infants with severe bronchiolitis
}

\author{
Florent Baudin ${ }^{1,2^{*}}$ (D), Guillaume Emeriaud ${ }^{3,4}$, Sandrine Essouri ${ }^{4}$, Jennifer Beck ${ }^{5,6,7}$, Etienne Javouhey ${ }^{1,2}$ and \\ Claude Guerin $8,9,10,11,12$
}

Dear Editor,

Though neurally adjusted ventilatory assist (NAVA) is known to improve patient-ventilator interactions in infants with bronchiolitis [1,2], its impact on respiratory muscles unloading has not previously been studied.

We conducted a secondary analysis (ethics committee approval CE_SRLF_18-48) of a prospective physiological study [3] which evaluated the impact of body positioning on work of breathing (WOB) in infants with severe bronchiolitis. Seven of the children included (median age 35 [27-63] days) had a respiratory recording during the transition from nasal continuous positive airway pressure (nCPAP; set at $7 \mathrm{cmH}_{2} \mathrm{O}$ [4]) to NAVA. Esophageal (Peso), gastric (Pga), and airway (Paw) pressures, as well as Electrical activity of the diaphragm (Edi), and flow were recorded simultaneously. Median NAVA level was set at $0.7[0.7-0.9] \mathrm{cmH}_{2} \mathrm{O} / \mu \mathrm{V}$ and median positive end expiratory pressure at 5 [4-7] $\mathrm{cmH}_{2} \mathrm{O}$. Twenty-five breaths during the last $2 \mathrm{~min}$ in nCPAP then during the first $2 \mathrm{~min}$ in NAVA were analyzed off-line. Metabolic cost of breathing was estimated by the Peso (PTPeso) and diaphragmatic (PTPdi) pressure time product, inspiratory effort by the Peso $(\Delta$ Peso) and diaphragmatic $(\Delta$ Pdi) pressure swings, and respiratory drive by the Edi swing ( $\triangle$ Edi). Data were expressed as median [IQR] and compared using Wilcoxon two-sample paired sign test. A $p$ value $<0.05$ was considered significant.

\footnotetext{
* Correspondence: florent.baudin@chu-lyon.fr

${ }^{1}$ Hospices Civils de Lyon, Hôpital Femme Mère Enfant, Réanimation

Pédiatrique, 59 Bd Pinel, F-69500 Bron, France

${ }^{2}$ University Lyon, Université Claude Bernard Lyon1, Ifsttar, UMRESTTE, UMR

T_9405, F-69373 Lyon, France

Full list of author information is available at the end of the article
}

As detailed in Table 1 and illustrated in Fig. 1, all indices of WOB (PTPeso, PTPdi, $\triangle$ Peso, $\triangle$ Pdi, Edi swing, and inspiratory time to total time ratio (Ti/Ttot)) decreased significantly in every child with NAVA as compared to nCPAP $(p<0.05$ in all instances), while the mean Paw was increased $(p<0.05)$.

Table 1 Comparison of physiological parameters between nasal continuous positive airway pressure and neutrally adjusted ventilatory assist

\begin{tabular}{llll}
\hline & nCPAP & NAVA & $p^{*}$ \\
\hline Ti/Ttot (\%) & $0.47[0.45-0.49]$ & $0.40[0.37-0.45]$ & 0.02 \\
Respiratory rate $(/ \mathrm{min})$ & $71[64-84]$ & $65[57-80]$ & 0.31 \\
Mean airway pressure $\left(\mathrm{cmH}_{2} \mathrm{O}\right)$ & $7.0[6.9-7.1]$ & $10.6[9.4-11.9]$ & 0.02 \\
$\Delta$ Edi $(\mu \mathrm{V})$ & $19[17-25]$ & $16[10-19]$ & 0.03 \\
Swing Peso $\left(\mathrm{cmH}_{2} \mathrm{O}\right)$ & $14[12-18]$ & $8[8-13]$ & 0.01 \\
Swing Pdi $\left(\mathrm{cmH}_{2} \mathrm{O}\right)$ & $14[13-15]$ & $10[9-10]$ & 0.02 \\
PTPeso/breath $\left(\mathrm{cmH}_{2} \mathrm{O} \mathrm{s}\right)$ & $4.7[3.4-6.1]$ & $2.1[1.9-3.7]$ & 0.02 \\
PTPdi/breath $\left(\mathrm{cmH}_{2} \mathrm{O} \mathrm{s}\right)$ & $4.2[3.9-4.4]$ & $2.6[2.5-2.8]$ & 0.02 \\
PTPeso/min $\left(\mathrm{cmH}_{2} \mathrm{O} / \mathrm{min}\right)$ & $365[237-429]$ & $162[139-226]$ & 0.02 \\
PTPdi/min $\left(\mathrm{cmH}_{2} \mathrm{O} \mathrm{s} / \mathrm{min}\right)$ & $298[256-354]$ & $157[151-199]$ & 0.02 \\
\hline
\end{tabular}

Data are expressed as median [interquartile range]

$n C P A P$ nasal continuous positive airway pressure, NAVA neutrally adjusted ventilatory assist, PEEP positive end expiratory pressure, $T i$ inspiratory time, Ttot total time, Peso esophageal pressure, Pga gastric pressure, Edi electrical activity of the diaphragm, PTP pressure time product *Wilcoxon two-sample paired sign test

In this physiological study, we report an improvement of respiratory unloading by adding a second level of pressure with NAVA in infants with severe bronchiolitis. WOB decreased immediately after switching to NAVA (Fig. 1), as reported previously in adults with obstructive lung diseases [5], and was associated with a lower neural drive and $\mathrm{Ti} / \mathrm{Ttot}$ ratio. 


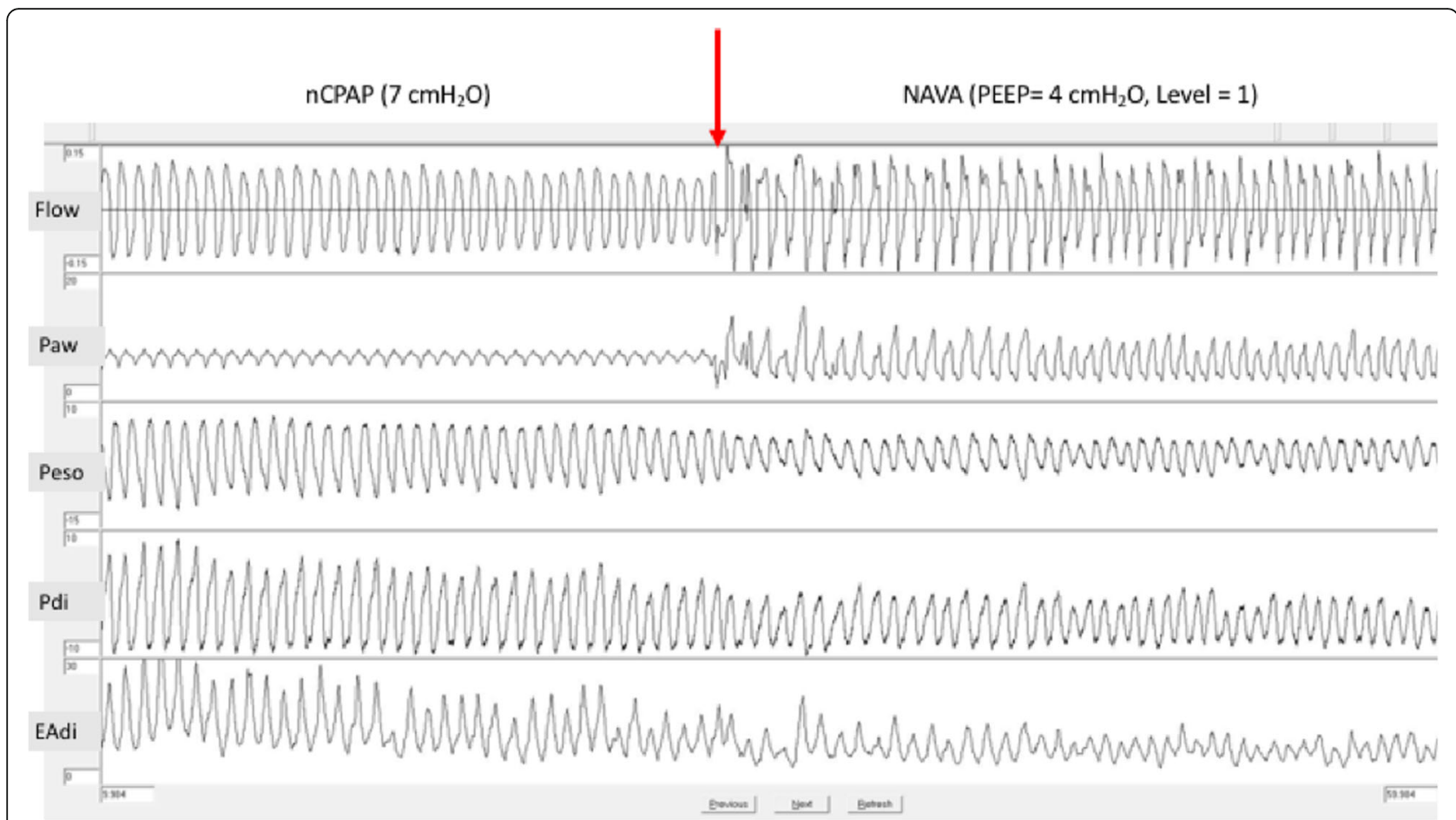

Fig. 1 Decrease of esophageal and trans-diaphragmatic pressure swing and Edi amplitude after switching to neurally adjusted ventilatory assist. The red arrow indicates the switch from nCPAP to NAVA. nCPAP, nasal continuous positive airway pressure; NAVA, neurally adjusted ventilatory assist; PEEP, positive end expiratory pressure; Paw, airway pressure; Peso, esophageal pressure; Pga, gastric pressure; EAdi, electrical activity of the diaphragm

This study has several limitations, including the small sample size, the short study period, the non-randomized order of recordings, and the non-standardized NAVA settings. However, the consistent, rapid, and large improvement in WOB-related indices observed in every infant is an important finding, especially considering the number of infants with severe bronchiolitis who may benefit from an improvement in non-invasive support. The findings support the need for further evaluation of the potential interest of NAVA to improve the efficiency of non-invasive support in infants with bronchiolitis.

\section{Abbreviations}

Edi: Electrical activity of the diaphragm; IQR: Interquartile range; NAVA: Neurally adjusted ventilatory assist; nCPAP: Nasal continuous positive airway pressure; Paw: Airway pressure; Peso: Esophageal pressure; Pga: Gastric pressure; PTP: Pressure time product; WOB: Work of breathing

\section{Acknowledgements}

We thank Philip Robinson (DRCl, Hospices Civils de Lyon) for critical revision of the manuscript.

\section{Funding}

The study was supported by a grant from the Fondation du Souffle et le Fonds de Dotation "Recherche en Santé Respiratoire", by a grant from the Association Lyonnaise de Logistique Post Hospitalière (ALLP), and by a grant from the the Groupe Francophone d'Urgence et de Réanimation Pédiatrique (GFRUP).

\section{Availability of data and materials}

The datasets generated and/or analyzed during the current study are not publicly available according to the French National Data Protection Commission (CNIL) but are available from the corresponding author on reasonable request.

\section{Authors' contributions}

FB and EJ conceived the study, analyzed and interpreted the data, and drafted the manuscript. GE, SE, JB, and CG analyzed and interpreted the data and revised the manuscript. JB and SE provided the technical support. All authors read and approved the final manuscript.

\section{Ethics approval and consent to participate}

The primary study was approved by the institutional review board (CPP SUDEST3- $n^{\circ} 2015-057 \mathrm{~B}$ ) and by the national medicines authority (ANSM-151048B32) and written consent was obtained from the parent(s) or guardian(s). The secondary analysis was approved by the ethical committee of the French intensive care society (CE_SRLF_18-48).

\section{Consent for publication}

$\mathrm{N} / \mathrm{A}$

\section{Competing interests}

FB has received speaking fees and non-financial support from Maquet Critical Care.

GE'S research program is supported by a research grant from the Fonds de recherche en santé du Québec. He also recently conducted a feasibility study of a new ventilator, which costs were supported by Maquet Critical Care.

JB has been reimbursed by Maquet Critical Care (Solna, Sweden) for attending several conferences; JB has participated as a speaker in scientific meetings or courses organized and financed by Maquet Critical Care; JB, through Neurovent Research, serves as a consultant to Maquet Critical Care. The following disclosure was agreed upon by University of Toronto, 
Sunnybrook Health Sciences Centre, St-Michael's Hospital, and the REBs of Sunnybrook and St-Michael's to resolve conflicts of interest: "Dr. Beck has made inventions related to neural control of mechanical ventilation that are patented. The patents are assigned to the academic institution(s) where inventions were made. The license for these patents belongs to Maquet Critical Care. Future commercial uses of this technology may provide financial benefit to Dr. Beck through royalties. Dr Beck owns 50\% of Neurovent Research Inc (NVR). NVR is a research and development company that builds the equipment and catheters for research studies. NVR has a consulting agreement with Maquet Critical Care."

The others authors declare that they have no conflict of interests.

\section{Publisher's Note}

Springer Nature remains neutral with regard to jurisdictional claims in published maps and institutional affiliations.

\section{Author details}

${ }^{1}$ Hospices Civils de Lyon, Hôpital Femme Mère Enfant, Réanimation Pédiatrique, 59 Bd Pinel, F-69500 Bron, France. University Lyon, Université Claude Bernard Lyon1, Ifsttar, UMRESTTE, UMR T_9405, F-69373 Lyon, France. ${ }^{3}$ Pediatric Intensive Care Unit, CHU Sainte-Justine, Université de Montréal, Montreal, QC, Canada. ${ }^{4}$ Department of Pediatrics, CHU Sainte-Justine, Université de Montréal, Montreal, QC, Canada. ${ }^{5}$ Keenan Research Centre for Biomedical Science, Li Ka Shing Knowledge Institute, St. Michael's Hospital, Toronto, ON, Canada. ${ }^{6}$ Institute for Biomedical Engineering and Science Technology (iBEST), Ryerson University and St-Michael's Hospital, Ontario, Canada. ${ }^{7}$ Department of Pediatrics, University of Toronto, Toronto, Ontario, Canada. ${ }^{8}$ Hospices Civils de Lyon, Hôpital de la Croix-Rousse, Médecine Intensive Réanimation, F-69004 Lyon, France. ${ }^{9}$ Université de Lyon, Université Claude Bernard Lyon 1, Villeurbanne, France. ${ }^{10}$ INSERM 955 - Eq13, Institut Mondor de Recherche Biomédicale, Créteil, France. ${ }^{11}$ Médecine Intensive-Réanimation, Grenoble, France. ${ }^{12}$ INSERM 1042 HP2, Grenoble, France.

Received: 4 February 2019 Accepted: 28 February 2019 Published online: 16 April 2019

\section{References}

1. Ducharme-Crevier L, Beck J, Essouri S, Emeriaud G. Neurally adjusted ventilatory assist (NAVA) allows patient-ventilator synchrony during pediatric noninvasive ventilation: a crossover physiological study. Crit Care. 2015;19:44

2. Baudin F, Pouyau R, Cour-Andlauer F, Berthiller J, Robert D, Javouhey E. Neurally adjusted ventilator assist (NAVA) reduces asynchrony during noninvasive ventilation for severe bronchiolitis. Pediatr Pulmonol. 2015;50:1320-7.

3. Baudin F, Emeriaud G, Essouri S, Beck J, Portefaix A, Javouhey E, et al. Physiological effect of prone position in children with severe bronchiolitis: a randomized cross-over study (BRONCHIO-DV). J Pediatr. 2019;205:112-4.

4. Essouri S, Durand P, Chevret L, Balu L, Devictor D, Fauroux B, et al. Optimal level of nasal continuous positive airway pressure in severe viral bronchiolitis. Intensive Care Med. 2011;37:2002-7.

5. Liu L, Xia F, Yang Y, Longhini F, Navalesi P, Beck J, et al. Neural versus pneumatic control of pressure support in patients with chronic obstructive pulmonary diseases at different levels of positive end expiratory pressure: a physiological study. Crit Care. 2015;19:244 